\title{
Serendipity in Context: Prioritised Contextual Browsing in Large-Scale Digital Libraries
}

\author{
David M. Weigl, lain Emsley, Kevin R. Page \\ Oxford e-Research Centre, Department of Engineering Science, University of Oxford, UK. \{firstname.lastname\}@oerc.ox.ac.uk
}

\begin{abstract}
In previous work we proposed a model of information-seeking behaviour in scholarly workset creation, combining and extending established models by Bates, Ellis, and Wilson to encompass strategies for scholarly research in large-scale information systems. However, this model simplifies contextual browsing, a key aspect of information seeking in large-scale information systems, as a single, holistic, strategy. Here, we extend this model with granular strategies for contextual browsing, defining new modes to characterise contextual browsing as combinations of these strategies, which we show to be consistent with serendipitous discovery as described by Makri et al. We study the properties of prioritised contextual browsing as a mechanism for implementing these strategies. We describe the Compage framework, a proof-of-concept implementation for prioritised contextual browsing of Linked Data resources, using Jaccard similarity for prioritisation. Extending Compage, we develop a simulation environment in which we investigate the utility of prioritised contextual browsing over a large-scale digital library dataset. Our simulation applies three strategies for the traversal of contextual metadata: reset, unprioritised, and prioritised. Results empirically demonstrate the advantages of prioritised contextual browsing, and that elements of serendipity can be identified and incorporated within our information-seeking model. In doing so, we evaluate our model's suitability for this scenario, yielding a more detailed understanding of the strategies and modes of behaviour underlying contextual browsing.
\end{abstract}

\section{KEYWORDS}

Information-seeking behaviour, digital libraries, contextual browsing, serendipitous discovery

\section{INTRODUCTION}

Large-scale digital libraries (DLs) provide unified access to massive sets of heterogeneous content. We have previously proposed a model of information-seeking behaviour in large-scale DLs (Weigl, Page, Organisciak, \& Downie, 2017) combining established models by Bates (1989), Ellis (1993), and Wilson (1997), extended with new strategies, classifications, and scopes of application. Our earlier model allows for more precise description of the challenges of supporting discovery, analysis, and scholarly use of the wealth of materials contained in DLs operating on massive scales. It supports use cases centred on worksets - machine-actionable research collections aggregating information resources alongside rich contextual metadata at various levels of granularity (Jett, Cole, Maden, \& Downie, 2016). The model incorporates contextual browsing as a coarse-grained strategy operating over routes for nuanced exploration and analysis embodied by this metadata, providing singular conditions for serendipitous discovery. Finer-grained modes of contextual browsing, highly relevant in the scenario, were unexplored.

In this paper, we more fully assess contextual browsing in three ways: through modelling; implementation; and simulation. In the first half, we describe a new model of contextual browsing, elaborating upon our previous model. We unpack contextual browsing — previously incorporated as a single, holistic strategy — into a family of component strategies. In combination, these give rise to different modes of contextual browsing, which we describe and harmonise with a framework of behavioural strategies for serendipitous discovery. In the second half, we discuss the application of our extended model at scale through applied prioritisation, and present the Compage framework, an implemented proof of concept of our approach. Finally, we evaluate the utility of prioritised contextual browsing, employing three synthetic browsing strategies within a computer simulation of information-seeking behaviour across a large collection of Linked Data.

\section{BACKGROUND: INFORMATION SEEKING AND SERENDIPITY IN LARGE-SCALE DIGITAL LIBRARIES}

Worksets are information constructs (Jett et al., 2016) supporting the requirements of scholars undertaking research in largescale DLs (Fenlon et al., 2014; Page et al., 2018). Their capabilities include: gathering documents within citable collections; supporting computational extraction of document-derived features for scholarly analysis; generating new descriptive information by inference over existing metadata; and linking complementary information from external sources.

Workset creation and refinement is particularly underserved by a view of information-seeking behaviour couched in singlequery sessions. Rather, workset building comprises iterative, evolving behaviours analogous to "berrypicking" (Bates, 1989). Our previous model of information-seeking behaviours for scholarly workset creation (Weigl et al., 2017) combined berrypicking strategies per Bates (1989), categories of information-seeking behaviour within the research process per Ellis (1993), and Wilson's modes of search (1997). This model classifies strategies as operating upon three scopes of application: content representations (e.g., images, text); content-derived metadata; and additional metadata derived via external (human or algorithmic) processes. 


\begin{tabular}{|c|c|c|c|c|c|c|c|c|}
\hline \multirow[b]{2}{*}{ Strategy } & \multicolumn{8}{|c|}{ Category of information-seeking behaviour (per Ellis, 1993) } \\
\hline & : & 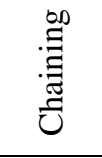 & $\begin{array}{l}1 \\
\text { b } \\
0 \\
0 \\
0\end{array}$ & 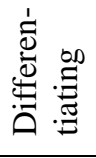 & 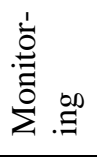 & 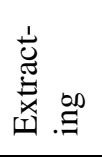 & 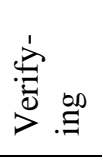 & 号 \\
\hline Contextual browsing & $\mathrm{S}$ & $\mathrm{A} / \mathrm{P} / \mathrm{S}$ & $\mathrm{A} / \mathrm{P}$ & A & & A & $\mathrm{A} / \mathrm{P}$ & $\mathrm{A} / \mathrm{P}$ \\
\hline Anchor specification & A & $\mathrm{A} / \mathrm{P}$ & $\mathrm{A} / \mathrm{P}$ & A & & A & & $\mathrm{A} / \mathrm{P}$ \\
\hline Target specification & & $\mathrm{A} / \mathrm{P}$ & $\mathrm{A} / \mathrm{P}$ & A & & A & $\mathrm{A} / \mathrm{P}$ & $\mathrm{A} / \mathrm{P}$ \\
\hline Profile specification & & $\mathrm{A} / \mathrm{P}$ & $\mathrm{A} / \mathrm{P}$ & A & $\mathrm{O}$ & & $\mathrm{A} / \mathrm{P}$ & \\
\hline Prioritisation & $*$ & $*$ & $*$ & $*$ & $*$ & $*$ & $*$ & $*$ \\
\hline
\end{tabular}

Modes of search-A: Active search, P: Passive search, O: Ongoing search, S: Serendipitous discovery (passive attention) *: Prioritisation is an ongoing process informed by and modulating behaviour across categories of information-seeking

The models of both Bates and Ellis incorporate chaining as a means of traversing an information domain where a searcher performs "successive leaps" (Bates, 1989) from document to document, following up references or records in a citation index. On the Web, more diverse types of inter-document association are available through hyperlinks; following links is classified by Choo, Detlor, and Turnbull (2000) as a chaining "Web move". In a library context, fixed-schema fields of catalogue records provide further means of inter-record reference and traversal. In DLs, mutable-schema contextual metadata contributed through ongoing user and system processes (Weigl et al., 2017) provide ever more connections; where they are expressed as Linked Data, chaining may occur between data entities, rather than documents, enabling more fine-grained explorations.

The creation of semantic layers of contextual metadata using Linked Data in DLs is subject to ongoing research (e.g., Fafalios, Holzmann, Kasturia, \& Nejdl, 2017; Page, Bechhofer, Fazekas, Weigl, \& Wilmering, 2017). RDF, a framework for the specification of Linked Data, associates contextual metadata with information entities by asserting \{subject, predicate, object $\}$ triples to specify directed relationships (predicates) between an entity (subject) and associated entities (objects). RDF-compliant systems operate on an open world assumption, meaning that the absence of a triple specifying a connection does not imply the absence of the connection, and that more triples can always be published, and new connections made.

These emergent connections provide prime conditions for serendipitous discoveries. Makri et al. build on empirical research into serendipitous information encounters among scholars (Makri \& Blandford, 2012) and creative professionals (Makri, Blandford, Woods, Sharples, \& Maxwell, 2014) to specify a set of information-system design suggestions to influence the likelihood of serendipitous encounters (Makri et al., 2014). Rather than proposing to "engineer" serendipity, a problematic notion given the inherent unexpectedness at its core, they identify human information behaviours that support the likelihood of serendipitous discovery by promoting the stages of the serendipity process: the making of a mental connection involving unexpected circumstance and insight; a projection regarding the connection's potential value, followed by actions taken to exploit it; culminating in a valuable, unanticipated outcome. These behaviours are identified from critical incident reports by participants reflecting on serendipitous information encounters in a semi-structured interview. Makri et al. (2014) detail the identified behaviours, providing corresponding recommendations for information-system design. The behaviours, numbered for reference, include:

1. Vary your routine, to provide more opportunities for making connections;

2. Be observant, to draw attention to unexpected aspects of the information environment;

3. Make mental space, reducing cognitive load to create contingencies for unexpected discovery;

4. Relax your boundaries, to avoid excluding valuable information entities due to overly narrow constraints;

5. Draw on previous experience, as a way of making and projecting the value of connections;

6. Look for patterns, in order to recognise unexpected alignments and extract meaning from them; and,

7. Seize opportunities, to exploit value from connections made.

\section{AN EXTENDED MODEL: STRATEGIES AND MODES OF CONTEXTUAL BROWSING}

\subsection{Contextual Browsing Strategies}

In this section we unpack contextual browsing, which we previously modelled (Weigl et al.; Section 2) as a single, unified strategy, into a family of new information-seeking strategies sharing key characteristics (Table 1). Each strategy enables or enacts chaining activities. The strategies make full use of the rich, open, inter-entity relationships expressed in the metadata of large-scale DLs, beyond inter-document traversal of reference lists or citation indices per Ellis and Bates. Our strategies are capable of connecting any addressable information resources unconstrained by scopes of application, interweaving content, 
Table 2: Modes of contextual browsing (modes indicated in bold)

\begin{tabular}{lll|ll|l} 
No anchor & One anchor & Multiple anchors & Target & Profile & Behaviours \\
\hline Recommendation & Entity description & Entity connections & No & No & $1,6,7$ \\
\hline Target description & Link discovery & Targeted prioritisation & Yes & No & $1,2,4,6,7$ \\
\hline Collaborative filtering & Contextualised review & Information filtering & No & Yes & $1,2,3,5,7$ \\
\hline Information filtering & Link discovery & Targeted prioritization & Yes & Yes & $1,2,3,4,5,6,7$
\end{tabular}

metadata derived from content during cataloguing, and contextual metadata supplemented by system or user processes. Together, they support traversals of contextual space, jumping between entities in collections on the web of data through emergent vectors of association.

Traversals are anchored to starting points (entities or worksets), providing a situated focus in contextual space. When not specified by the user (anchor specification, Table 1), the system must situate the traversal, e.g. by automated recommendation. Traversals may be constrained and guided according to defined entities and inter-entity relationships (target specification). This reduces cognitive load, at the cost of withholding unanticipated, potentially valuable connections from user attention. Prioritisation, applying profile specification according to user value projections or through algorithmic measures, further constrains the traversal space. Profiles combine user-specified "deferred query" expressions of interest (personalisation) with interaction history, e.g. by reference to the user's workset collection. Where these strategies guide the user to unanticipated locations in the collection, this provides the potential for serendipitous discovery per Makri et al. (as defined in Section 2).

\subsection{Modes of Contextual Browsing as a Means to Serendipitous Discovery}

Combinations of contextual browsing strategies give rise to new modes of contextual browsing (Table 2). Each combination is consistent with a number of serendipity-influencing information behaviours (Section 2) ${ }^{1}$ :

- Recommendation. In the absence of specified anchors, targets, and user profiles, a system is limited to recommending potential starting points for contextual browsing. These may be extracted algorithmically (e.g., based on popularity) or by curation. Wikipedia's "article of the day" is an example of such an approach.

- Entity description. Browsable contextual views presented over anchoring entities in a result set provide a tight integration of search and browsing behaviour, supporting behaviour 1: Vary your routine. Where an entity is selected as an anchor, its entity description provides a view of associated contextual metadata.

- Entity connections. Where multiple entities or worksets are specified as anchors, a view describing similarities and notable differences among their descriptions supports serendipity behaviour 6: Look for patterns.

- Target description, and Link discovery. Where a target entity or workset is specified, a target description details the target's contextual metadata. With an entity selection, link discovery describes connections between the selected entity and target(s). These modes support behaviours 4: Relax your boundaries, providing a means of alerting users to related workset items during search or browsing; and 6: Look for patterns, revealing connections between selected and targeted entities.

- Targeted prioritisation. Where multiple anchors are specified alongside a target, prioritised contextual description and filtering actions can be undertaken by reference to weightings (e.g., similarity metrics) relative to the target.

- Collaborative filtering. When profile information is available by reference to a user's workset history, or by specification of relevance criteria (personalisation), a system can draw attention to entities selected by other users with comparable profiles, supporting behaviour 2: Be observant.

- Contextualised review and Information filtering. A specified anchor can be associated with profile information on previous interactions, supporting behaviour 5: Draw on previous experiences. Multiple specified anchors can be prioritised accordingly, using profile-based information filtering approaches (Belkin \& Croft, 1992). These modes support behaviours 3: Make mental space by integrating categorization into the information environment, supporting reflection as information is acquired; and 5: Draw on previous experiences, providing a mechanism for the discovery of unexpected connections between information under current, and previous, consideration, allowing users to review and revisit information in the light of new contextual connections.

- When a target is specified in the presence of profile information this provides further opportunities for Information filtering, Link discovery, and Targeted prioritisation, surfacing connections between anchoring entities, specified targets, previously encountered information, and entities encountered by other similarly interested users.

\footnotetext{
${ }^{1}$ Note that behaviour 7, Seize opportunities, is implicitly supported through the use of worksets for capture, storage, organization, and annotation of information throughout the information-seeking process.
} 
In the remainder of this paper, we consider the practical application of the new contextual browsing strategies introduced above, by: outlining an approach toward applied prioritisation in order to manage the massive quantities of contextual information available in large-scale DLs (Section 4); implementing a proof-of-concept framework and user interface realizing prioritized contextual browsing (Section 5); and conducting a simulation study to empirically validate the utility of prioritisation in contextual browsing over a large dataset (Section 6).

\section{APPLIED PRIORITISATION FOR CONTEXTUAL BROWSING AT SCALE}

Worksets provide a foundation for scholarship using prioritised contextual browsing by gathering the necessary information resources and contextual metadata within persistent, addressable collections. Consider the case of an academic informationseeker finding resources on Bede the Venerable, an English 7th-century monk of St. Peter and St. Paul in the Kingdom of Northumbria, modern day Jarrow, Tyne and Wear. Interested in Roman Catholic biblical scholars, the user starts a workset containing Bede, and undertakes contextual browsing anchored upon Bede's record to discover further relevant information.

Bede is associated, via contextual relationships, with a large number of categories ${ }^{2}$. Some of these are highly relevant to the researcher's information need: Bede is categorised as a Benedictine biblical scholar; a 7th-century theologian; a Christian hagiographer. Others are less immediately applicable: Bede as an Anglo-Saxon poet; a chronologist; a trope theorist. Further categories are at best incidental to the topic at hand: Bede as a doctor, a person from Jarrow, a living thing. This example illustrates the need for information reduction in large-scale contextual browsing, and the utility of discerning by use case: consider the alternate priorities of a researcher establishing a prosopography of 7th-century Jarrow.

Unless specified as profile information, a user's interests and information needs are extrinsic to the data available in a DL. Through worksets, prioritised browsing can nevertheless be provided by prioritising according to computed contextual similarities with existing workset members. Here, similarity acts as a proxy for relevance to a topic captured by the workset.

We propose Jaccard distances computed by set operations over contextual descriptors - the predicates and objects associated with a given subject entity in Linked Data (RDF) datasets - as a meaningful measure of contextual similarity. Entities are compared by dividing the intersection of their associated \{predicate, object \} sets by their union. These comparisons are performed iteratively between each entity under consideration, and the collection of neighbouring entities available for subsequent navigation. This provides a data-intrinsic means of prioritisation for contextual browsing.

\section{REALISING APPLIED PRIORITISATION AS A SPACE FOR SERENDIPITY}

\subsection{The Compage Framework}

Compage $^{3}$, a new prototyping framework for prioritised contextual browsing, demonstrates the feasibility of our model and provides a platform for its empirical exploration. Compage is dataset-agnostic, capable of providing contextual views over any specified RDF graphs and constrained set of user journeys.

The framework maintains a collection of user-associated worksets. These can be created or extended via a set of panes presenting views of contextual metadata associated with entities in a result set, including: Entity descriptions providing comprehensive listings and summaries of the RDF predicates and objects associated with selected entities (Figure 1, top); Similarity views providing access to similarities and differences between selected and targeted entities (Figure 1, bottom); and Entity connections providing statistical summaries of the preponderance of different contextual metadata items within specified worksets, the user's workset collection, and the wider dataset. Each view provides traversal mechanisms supporting prioritised contextual browsing among the collection.

Workset targets may be specified to accommodate Link discovery. "Drilling-down" into the discovered connections, a user can explore contextual similarities from entities within the result set to entities in the user's workset collection (serving here as profile information), or with the wider dataset, providing a controlled means of broadening a search. Contextual information associated with a given entity may have numerous semantic relationships with different entities in the dataset. For instance, a date serving as 'birth date' for one entity may function as a publication, flourished, or death date for others. Compage enables summary and traversal of such values according to their semantic use context, realising a means of serendipitous discovery of new connections. During traversal, encountered entities may be added to the result set on user request. Similarities are recalculated after workset modification, providing the potential for further discoveries surfaced in the shifting contextual landscape.

To illustrate, consider an application of Compage targeting DBPedia (Lehmann et al., 2015), a Linked Data publication of structured Wikipedia information. A researcher interested in studying Benedictine writers might incorporate our protagonist

\footnotetext{
${ }^{2}$ Illustrative examples from the Bede the Venerable DBPedia resource: http://dbpedia.org/resource/Bede

${ }^{3}$ Available at https://github.com/oerc-csi/compage
} 


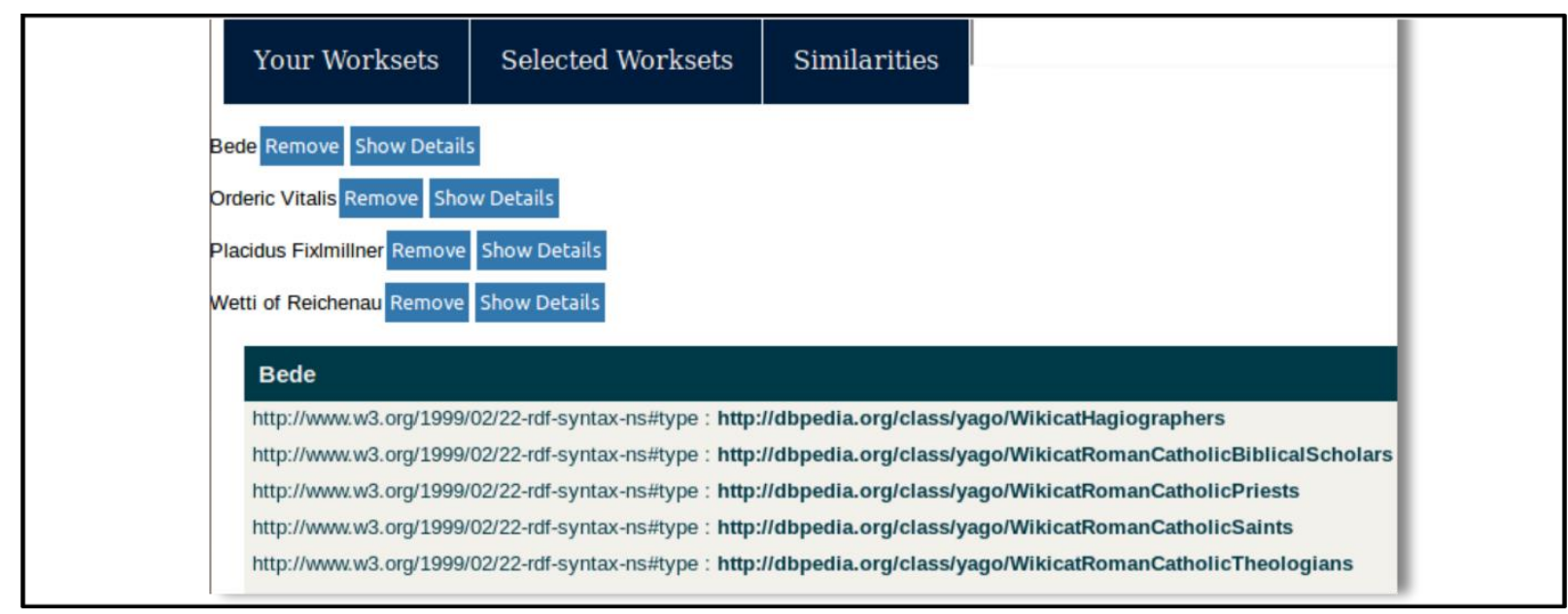

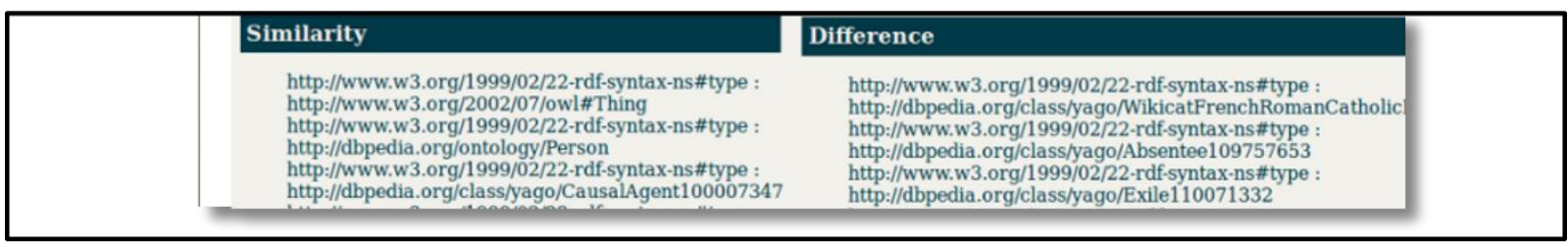

Figure 1: Top: Compage result set of Benedictine scholars, with entity descriptions for Bede the Venerable. Bottom: Entity connections pane with (a subset of) contextual similarities and differences between Bede and Orderic Vitalis

the Venerable Bede into a result set — by typing his name into a rudimentary search interface, or by loading a pre-specified workset of Benedictine scholars. Upon requesting an entity description ("Show Details"), the user discovers information relating to Bede's dates of birth, death, as well as the date on which he was canonised. Other entities associated with these date relationships can be retrieved from the current result set, a specified workset, or within the wider data. Alternatively, the user may discover from the entity description that Bede was canonised by Pope Leo XIII, and traverse to this associated entity to request its (Pope Leo's) associated entities. In either case, Jaccard similarities can be calculated to limit the set of retrieved entities to those similar to the ones currently under consideration, supporting discovery while constraining information load.

Compage employs a client/server architecture that uses client requests to parameterise SPARQL queries. Queries and similarity calculations are executed on the server via a triplestore ${ }^{4}$, reducing client load. A JavaScript client presents the user interface, communicating requests to the server and caching data currently under consideration in order to minimise recomputation.

\subsection{Model Conformance}

Compage implements aspects of our extended model (Section 3) and notions of applied prioritisation (Section 4):

1. Anchor specification within a rudimentary search result set;

2. Contextual views over entity and workset descriptions;

3. Inter-entity similarity and difference listings;

4. Directed Jaccard prioritisation of contextual space;

5. Target and relation specification, enabling inter-entity traversals according to related contextual descriptions;

6. Constrained traversals targeting worksets in the user's collection;

7. Instantiation and refinement of worksets to include relevant entities as they are discovered (extraction).

As such, Compage demonstrates the feasibility of implementing the approaches discussed in this paper.

The framework does not currently implement other DL information-system affordances, such as prioritisation according to popularity with other users sharing related interests (e.g., for collaborative filtering). However, its modular, dataset-independent

\footnotetext{
${ }^{4}$ Compage may be configured to query any triplestore exposing a SPARQL 1.1-compliant endpoint. It has been tested with Virtuoso and Blazegraph.
} 
design is intended to support such applications in the future by treating crowd-sourced data, and other emergent metadata streams, as yet more context to be explored.

\section{SIMULATING SCHOLARLY CONTEXTUAL BROWSING AND SERENDIPITOUS ASSOCIATIONS}

Scholarly contextual browsing is a time-intensive undertaking. Aside from the time required for careful application of insight, the problem is one of combinatorial explosion inherent in graph structures. Consider the example of a family tree; moving backwards, ancestor by ancestor, through successive generations from a single person doubles the search space with every hop, from the person's two parents, to the four grandparents, eight great grandparents, and so on. This problem is compounded in a DL context, where each node is connected to a potentially large number of neighbours. Consider again the Venerable Bede; he has direct connections to 133 neighbouring graph entities; in turn, these are connected to 364 entities, Bede's neighbours once removed. There are 1,878 neighbours twice removed, and 38,948 neighbours thrice removed. The Compage framework assists users in constraining and directing their contextual browsing through this space by prioritisation according to similarity. Even so, longitudinal user studies are required to observe information seekers engaging in contextual browsing in such a scenario; we reserve such studies for future work. Here, we instead simulate these strategies for traversals across a large dataset to evaluate the utility of prioritised over unprioritised contextual browsing - the proposition at the heart of Compage. We treat similarity as a simplified proxy measure for topical relevance, where the topic is approximated by the membership of a seed

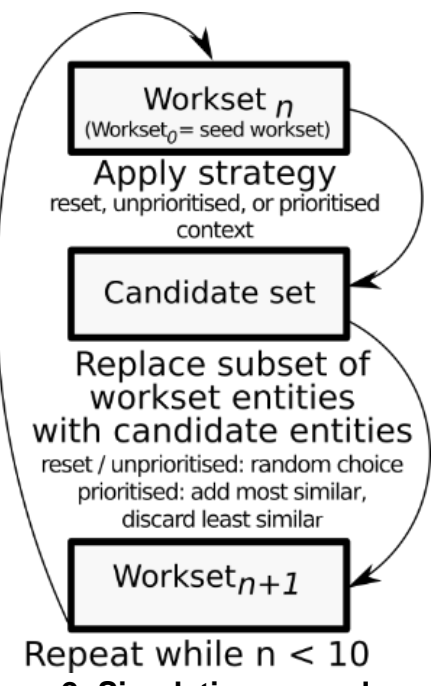

Figure 2: Simulation procedure workset. The goal of the simulated agent is to traverse iteratively through contextual space, incorporating new information while maintaining similarity (as relevance) both to the seed workset starting the traversal, and to the immediate predecessor workset at each iterative hop.

\subsection{Dataset and Seed Worksets}

Our simulation targets DBPedia (Lehmann et al., 2015). This Linked Data publication of structured Wikipedia information exhibits comprehensive coverage, a heterogeneous nature, and a high degree of interconnectedness. Thus, it provides an ideal proving ground for contextual browsing approaches. The simulation could equally target any RDF collection of large-scale DL metadata; the corresponding $\mathrm{R}$ script ${ }^{5}$ can be pointed at any SPARQL endpoint. We have chosen two seed worksets for our simulation: Seed1: a subset of 50 entities randomly selected from the category Roman Catholic theologians (which contains 468 members on DBPedia); and Seed2: the more specialised subcategory of Benedictine Writers, which contains 13 members.

\subsection{Simulated Strategies}

Our agents employ one of three synthetic contextual browsing strategies, applying categories of information-seeking behaviour and (simulated) modes of search outlined in our extended model (Section 3). Reset context gathers new entities by randomly sampling the contextual space; an iterative, memoryless re-starting (per Ellis; see Table 1). This provides a baseline featuring neither chaining (following connections) nor differentiating (prioritising according to similarity), simulating a perpetual serendipity seeker, akin to a user making obsessive use of Wikipedia's "random article" feature in the hope of encountering relevant information - though lacking human insight to know the best entities to add to the current workset as they are encountered. Unprioritised context randomly traverses connections from entities in the current set. On each iteration this agent "follows its nose" through the contextual neighbourhood, chaining without differentiating, by adding a random selection of neighbouring entities to the workset at each step. This simulates a purely "passive searcher" per Wilson, constantly starting new search tangents based on previous outcomes. Prioritised context traverses between connected entities, prioritising by Jaccard similarity. This corresponds to chaining with differentiating according to similarity. This strategy simulates an "active searcher" per Wilson, pursuing an information need of topical relevance (here, similarity) to the current workset.

\subsection{Procedure and Testing}

Our simulation proceeds by iterative application of the simulated strategies (Figure 2), starting from the previous iteration's workset, and simulating contextual browsing to produce a new workset at each step. The simulation is run twice, either: maintaining a steady workset size across iterations, replacing $\frac{1}{5}$ of the preceding workset at each step; or narrowing the workset

\footnotetext{
${ }^{5}$ Available at https://github.com/oerc-csi/contextualBrowsingSim
} 


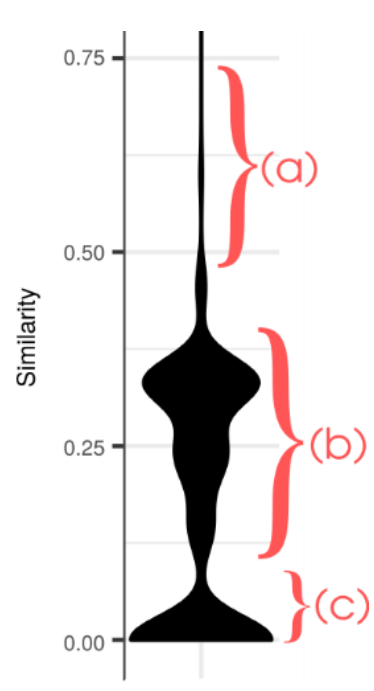

Figure 3: Example similarity distribution between a workset and its predecessor

across iterations by $\frac{1}{5}$ of the preceding workset's size (incorporating $\frac{1}{10}$ new entities at each step). This simulates the distinct behaviours of open-ended exploration (approximating a steady state), or narrowing down toward the end of a research process.

Workset-to-workset distances comprising the Jaccard similarities (Section 4) are calculated between each entity in one workset and all entities in the other. These distances are computed between each workset and its predecessor; and between each workset and the corresponding seed (Seed1 or Seed2). From these we produce a distribution of distances, which we refer to as a similarity distribution. Figure 3 shows an example similarity distribution visualised as a violin plot (a smoothed histogram reflected along the y-axis) where the width at any point corresponds to the proportion of inter-workset comparisons in the distribution sharing the corresponding similarity value. In the example, the narrow tail (a) indicates the presence of a small number of highly similar entities. The body of the distribution (b) characterises the range of typical (non-zero) similarity values present in the distribution: here, the median nonzero score is 0.33 , slipping to .23 when including zero values. Finally, the foot of the distribution (c) indicates a large number of zero-similarity comparisons in the example.

We apply two-sample Kolmogorov-Smirnov tests between pairs of similarity distributions to test whether they share an underlying continuous distribution, or differ significantly:

Test 1: Do similarity distributions between each workset and the corresponding seed workset differ significantly across the simulated strategies? Null hypothesis 1: The produced worksets are equally similar to their seeds.

Test 2: Do similarity distributions between each workset and its predecessor differ significantly across the simulated strategies? Null hypothesis 2: The strategies produce worksets that are equally similar to their predecessor, at each step.

Test 3: Do similarity distributions between each workset and its predecessor differ significantly within each simulated strategy's iterations? Null hypothesis 3: There is no divergence in similarity distributions across iterative applications of each strategy.

We apply Šidák correction (1967) to account for multiple comparisons (declaring significance at $p<.0017$ ).

\subsection{Visualisation and Interpretation}

The resulting similarity distributions are visualised in Figures 4 (steady-state) and 5 (narrowing-down). Within each figure, the top two panels represent simulated traversals starting at Seed1 (Roman Catholic theologians), and the bottom two panels starting at Seed2 (Benedictine writers). Each plot illustrates the similarity distributions generated at each iterative step, for each of the three synthetic strategies distinguished by colour: prioritised context (green); unpriorisited context (orange); and reset context (purple). Each plot contains similarity distributions against the seed workset $(a)$, and similarity distributions against the predecessor workset $(b)$. The $\mathrm{y}$-axis of $(b)$ in each figure is inverted; proximity to the division line between $(a)$ and $(b)$ indicates lower similarity. Inspecting the plots across incremental iterations (from left to right), the changes in similarity distributions as each strategy unfolds may be visualised. A "sagging" of the distribution shape toward the division line (similarity = 0) indicates a tendency toward diminishing returns over successive iterations; whereas a retention of shape, or a tendency away from the line, implies that the strategy is successful at continuing to discover similar entities as the simulation proceeds.

Within each simulated step the prioritised context strategy, followed by unprioritised context, consistently produces the most similar distributions, both to the seed $(a)$ and to predecessor $(b)$ worksets. The reset context strategy consistently produces the least similar distributions and the most zero-similarity comparisons. However, it also occasionally produces outliers (manifested as narrow tails in the similarity distributions) that match or exceed the best scores produced by the other two strategies. The statistical reasoning behind these observations is as follows.

\subsection{Statistical Analysis}

For the steady state simulation, our two-sample Kolmogorov-Smirnov tests indicate that each of the three strategies produce significantly different similarity distributions at every step when comparing each iterative workset to the seed $(a)$, with one exception (Seed2, unprioritised vs. reset context at iteration 6). Overall, this strongly suggests that Null Hypothesis 1 can be rejected for the steady state simulation. 

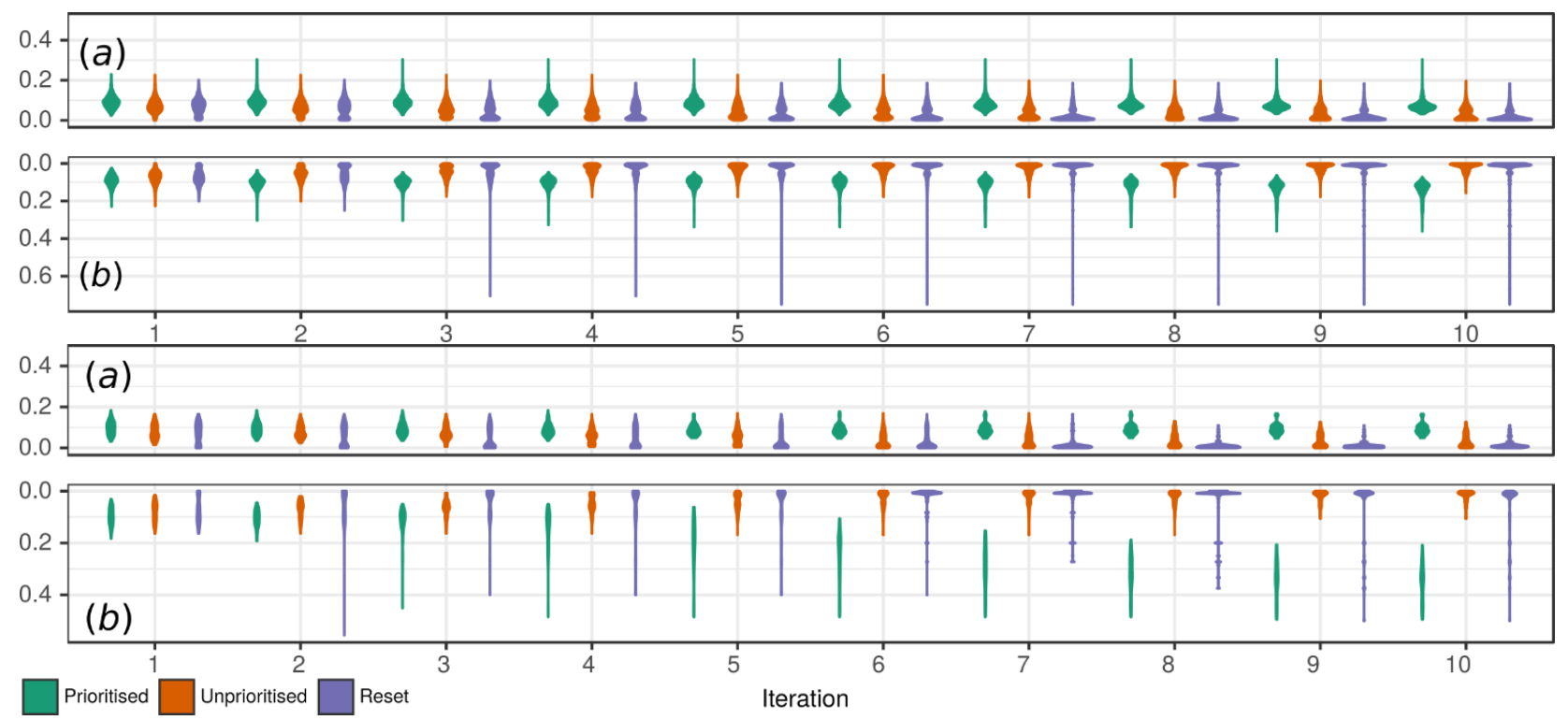

Figure 4: Similarity distributions generated by the simulation at steady workset size (replacing $\frac{1}{5}$ at each iteration). Top two panels: Iterations of Seed1, Roman Catholic theologians. Bottom two panels: Iterations of Seed2, Benedictine writers. In each iteration, similarities are computed between the generated workset and: (a) the corresponding seed workset, and $(b)$ the immediate predecessor produced by the same strategy.
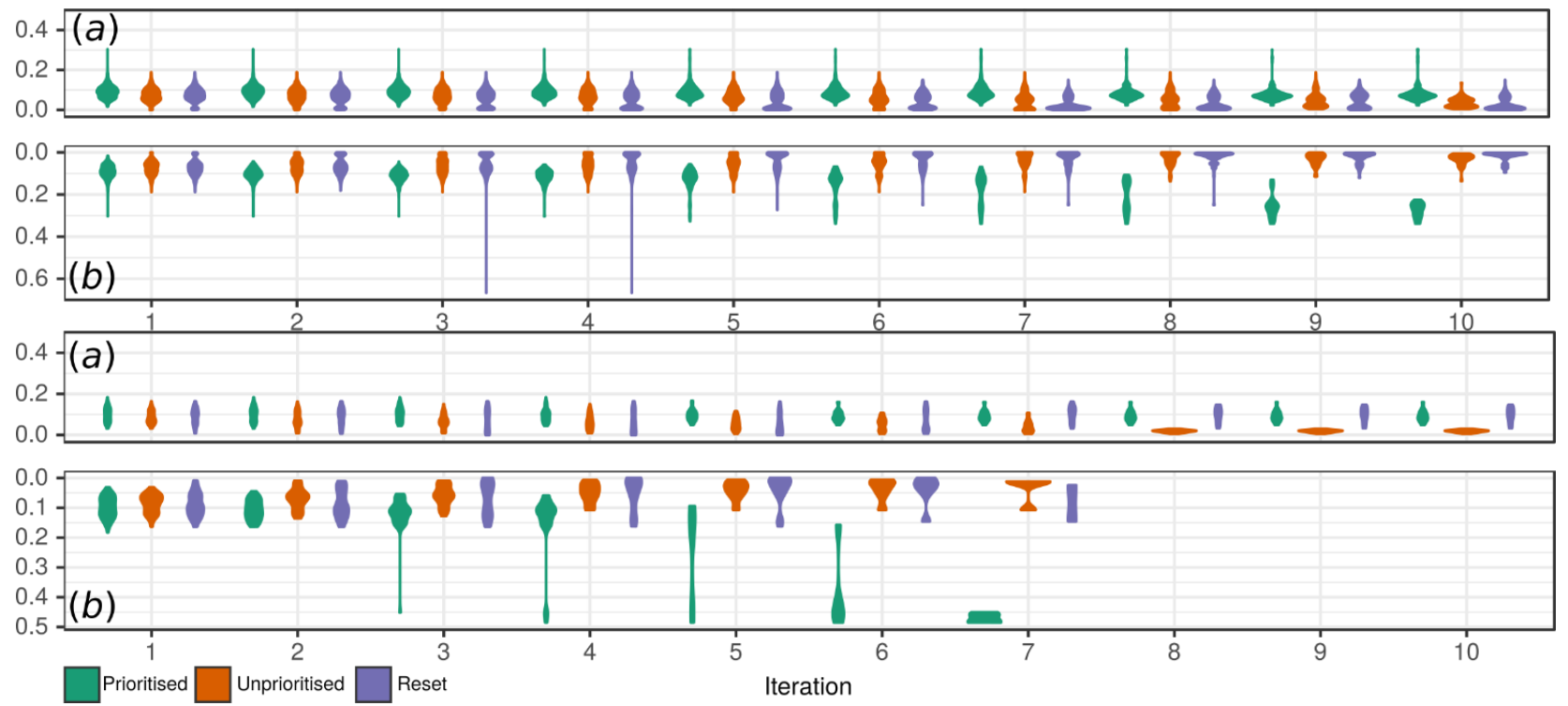

Figure 5: Similarity distributions generated by the simulation at shrinking workset size (narrowing by $\frac{1}{5}$ at each iteration). See also the caption for Figure 4, above. N.b. Workset size is 1 by the $7^{\text {th }}$ iteration-hence the missing values in the lower panel (self-similarities are excluded from analysis).

For the narrowing simulation, the results for $(a)$ are the same for Seed1, where each strategy produces significantly different similarity distributions at each iteration, with one exception (unprioritised and reset context, iteration 1). For Seed2, there are several exceptions: all three strategies exhibit only insignificant differences in their similarity distributions on iterations 1 and 2 , with the unprioritised and reset context strategies remaining insignificantly different for iterations 3,4 , 5, and 6 . This is explicable by the small size of the workset (13 members) and the narrowing nature of the simulation, meaning that only a small proportion of the data is modified in each step.

Interestingly the prioritised strategy separates itself early on from the reset context strategy (random baseline), suggesting that prioritised browsing provides more relevant discoveries even in small-scale explorations. Both the prioritised and reset context strategies exhibit insignificant differences for the same seed workset in iteration 7, 8, 9, and 10. This is because the narrowing process for this workset arrives at a membership of 1 by the 7 th iteration, which happens to be equidistant in both cases.

For similarity to the predecessor $(b)$, our results indicate significant differences in distribution across all strategies for each simulated step in the steady state simulation. We reject Null Hypothesis 2. 
In the narrowing simulation, results for $(b)$ are again the same for Seed1, with two exceptions (unprioritised and reset context, iterations 1 and 9). Again they are divergent for the smaller Seed2, where differences in predecessor similarity are insignificant between all strategies for iterations 1 and 2, and between unprioritised and reset context for all remaining steps. Again, prioritised browsing distinguishes itself after a few iterations, even in this small-scale scenario. The differences become insignificant among all strategies from the 7th iteration onward, again as an artifact of the narrowed workset's single-entity membership.

Results are mixed comparing similarity distributions of worksets to their predecessors $(b)$ within each strategy's outcomes. For the steady state Seed1 simulation, the following comparisons did not yield statistically significant differences in distribution:

- Reset context: Similarity to predecessor at steps 5 and 6; and at steps 9 and 10.

- Unprioritised context: Similarity to predecessor at steps 6 and 7; 8 and 9; and, 9 and 10.

- Prioritised context: Similarity to predecessor at steps 3 and 4; 4 and 5; 5 and 6; and, 6 and 7.

Comparing similarity distributions of worksets to their predecessor within each strategy in Seed2 of the steady state simulation, only the following comparisons yielded statistically significant differences:

- $\quad$ Reset context: Steps 2 and 3.

- Unprioritised context: Steps 6 and 7.

- Prioritised context: Steps 5 and 6;6 and 7; 7 and 8 .

Results for the narrowing simulation were comparably mixed. For Seed1, only within-strategy predecessor comparisons between steps 1 and 2, and 9 and 10 of the prioritised strategy, steps 2 and 3 of the unprioritised strategy, and steps 2 and $3 ; 3$ and 4 ; and 4 and 5 of the reset context strategy differing significantly. For Seed2, there were no significant within-strategy predecessor comparisons among any of the strategies.

\subsection{Discussion: Contextual Browsing as a Vehicle for Serendipitous Discovery}

In the context of our extended model (Section 3), grounded in Wilson's modes of search (Section 2), our simulation informs expectations on the utility of contextual browsing for human information seekers. Each workset's similarity to its seed provides a proxy for relevance outcomes of active search behaviour, where the seed workset represents a sample of results fulfilling an actively-pursued information need. Each workset's similarity to its predecessor is analogous to instances of passive search, where the pursuit of information results in the incidental acquisition of relevant information not subject to the active search.

Our simulated strategies can be viewed as agents pathologically applying active and passive search, and passive attention behaviours (the last of which involves serendipitous discovery of information outside of an information-seeking context). The prioritised context agent ruthlessly pursues its information need ("find other entities similar to this one"); the unprioritised context agent wanders amongst tangential connections; whereas the reset context agent constantly finds itself disconnected and memoryless within the information space, while occasionally chancing upon highly relevant entities.

Of course, separately incarnating the modes of search is an artificial exercise: human information seeking employs each mode simultaneously, particularly outside of single-query sessions, as in the continuous berrypicking of workset refinement. Nevertheless, our simulation results empirically demonstrating the potential value of prioritised contextual browsing in DL systems. They further demonstrate that the prioritised context strategy retains seed workset similarity, and gradually improves upon predecessor similarity over the course of the simulation. In other words, prioritised context rewards continued exploration for both active and passive search outcomes, whereas unprioritised context demonstrates a tendency toward diminishing returns.

Although random, the reset context strategy occasionally yields highly relevant results by pure serendipity. While clearly not a sensible mechanism for primary navigation of an information resource - our results show the overall similarity distributions produced by reset context are poor - this supports the utility of stochastic elements as secondary navigation vectors, per Makri et al.'s relaxing boundaries and making mental space serendipity behaviours.

\section{CONCLUSIONS}

In this paper we have extended our earlier model for information seeking in large-scale digital libraries with new strategies and modes of contextual browsing, showing their consistency with behaviours supporting serendipitous discovery set out by Makri et al. We have described how contextual browsing requires prioritisation to remain feasible in large-scale scenarios, and have proposed the application of contextual similarity metrics to this purpose. Compage, a prototype for prioritised contextual browsing over RDF datasets, illustrates the suitability of our model through implementation; furthered by automated assessment of our strategies in a simulated extension of the framework. This simulation has empirically demonstrated the utility of prioritisation in a study comparing prioritised, unprioritised, and reset context strategies for contextual browsing.

We believe that prioritised contextual browsing, supporting and combining both active and passive search behaviours, creates a space in which users can benefit from structured information while encouraging the likelihood of serendipitous discoveries. 
Serendipity cannot be "engineered", as Makri et al. highlight, and we do not claim to have done so here. Unexpectedness is, after all, the key precept of serendipity. Our results do capture occasions of "pure" serendipity, at least within the synthetic confines of our simulation, in the rare gems uncovered by our - otherwise pathological - reset-context strategy.

Longitudinal user studies of scholars pursuing real information needs are required to more fully understand human contextual browsing behaviour. These future studies must use modern information systems capable of exposing mutable navigation vectors along contextual metadata - through the theoretical, applied, and experimental contributions in this paper, we hope to have provided a framing for such future work.

We arrive at a number of conclusions concerning contextual browsing of large-scale DLs: $i$ ) that a theoretical conception of this process as a holistic activity occludes considerable complexities relating to motivations, information affordances, and system and user behaviours, which continuously shift as information-seeking takes place; ii) that prioritisation is a necessary component of meaningful contextual browsing in large-scale scenarios; and iii) that the rich contextual metadata available in large-scale DLs provide valuable vectors of discovery for both actively pursued and serendipitously encountered information, above and beyond the search and browsing behaviours afforded by traditional, limited-schema library metadata.

\section{ACKNOWLEDGEMENTS}

This work was supported by grant \#41500672 from the Andrew W. Mellon Foundation; and by Fusing Audio and Semantic Technologies for Intelligent Music Production and Consumption funded by the UK Engineering and Physical Sciences Research Council (EPSRC) under grant number EP/L019981/1. Conclusions and opinions expressed are those of the authors and are not necessarily shared by the sponsor.

\section{REFERENCES}

Bates, M. J. (1989). The design of browsing and berrypicking techniques for the online search interface. Online review 13(5), 407-424.

Belkin, N. J. \& Croft, W. B. (1992). Information filtering and information retrieval: Two sides of the same coin? Commun. ACM 35, 12, 29-38.

Choo, C. W., Detlor, B., \& Turnbull, D. (2000). Information seeking on the Web: An integrated model of browsing and searching. First Monday 5(2).

Ellis, D. (1993). Modeling the information-seeking patterns of academic researchers: A grounded theory approach. The Library Quarterly, 469-486.

Fafalios, P., Holzmann, H., Kasturia, V., \& Neijdl, W. (2017). Building and querying semantic layers for Web archives. In Proceedings of the 17 th ACM/IEEE-CS on Joint Conference on Digital Libraries, Vol. 2017.

Fenlon, K., Senseney, M., Green, H., Bhattacharyya, S., Willis, C., \& Downie, J. S. (2014). Scholar-built collections: A study of user requirements for research in large-scale digital libraries. In Proc. Association for Information Science and Technology 51(1), 1-10.

Jett, J., Cole, T. W., Maden, C., \& Downie, J. S. (2016). The HathiTrust Research Center Workset Ontology: A descriptive framework for non-consumptive research collections. Journal of Open Humanities Data 2.

Lehmann, J., Isele, R., Jakob, M., et al. (2015). DBPedia - A large-scale, multilingual knowledge base extracted from Wikipedia. Semantic Web 6(2), 167-195.

Makri, S. \& Blandford, A. (2012). Coming across information serendipitously - part 1: A process model. Documentation 68(5), 684-705.

Makri, S., Blandford, A., Woods, M., Sharples, S., \& Maxwell, D. (2014). "Making my own luck": Serendipity strategies and how to support them in digital information environments. In Journal of the Association for Information Science and Technology 65(11), 2179-2194.

Page, K. R., Bechhofer, S., Fazekas, G., Weigl, D. M., \& Wilmering, T. (2017). Realising a layered digital library: exploration and analysis of the Live Music Archive. In Proc. Joint Conference on Digital Libraries, IEEE, 1-10.

Page, K. R., Jett, J., Cole, T. W., Kudeki, D., Bainbridge, D., Organisciak, P., \& Downie, J. S. (2018). Worksets expand the scholarly utility of digital libraries. In Proc. 18th ACM/IEEE on Joint Conference on Digital Libraries (pp. 371-372). ACM.

Šidák, Z. (1967). Rectangular confidence regions for the means of multivariate normal distributions. The American Statistical Association 62(318), 626-633.

Weigl, D. M., Page, K. R., Organisciak, P., \& Downie, J. S. (2017). Information seeking in large-scale digital libraries: Strategies for scholarly workset creation. In Proceedings of the Joint Conference on Digital Libraries, IEEE, 1-4.

Wilson, T. D. (1997). Information behaviour: an interdisciplinary perspective. Information Processing \& Management, 33(4), 551-572.

$81^{\text {st }}$ Annual Meeting of the Association for Information Science \& Technology | Vancouver, Canada | Nov. 10 - 14, 2018 